\title{
X-ray probes of magnetospheric interactions with Jupiter's auroral zones, the Galilean satellites, and the Io plasma torus
}

\author{
R.F. Elsner ${ }^{\text {a,* }}$, B.D. Ramsey ${ }^{\text {a }}$, J.H. Waite Jr. ${ }^{\text {b }}$, P. Rehak ${ }^{\text {c }}$, R.E. Johnson ${ }^{\text {d }}$, J.F. Cooper ${ }^{\mathrm{e}}$, \\ D.A. Swartz ${ }^{\mathrm{f}}$ \\ a NASA Marshall Space Flight Center, XD 12, Huntsville, AL 35812, USA \\ ${ }^{\mathrm{b}}$ Department of Atmospheric, Oceanic, and Space Sciences, University of Michigan, 2455 Hayward St., Ann Arbor, MI 48109-2143, USA \\ ${ }^{\mathrm{c}}$ Instrumentation Division 535 B, 20 Technology Street, Brookhaven National Laboratory, Upton, NY 11973, USA \\ d Engineering Physics, University of Virginia, Charlottesville, VA 22901, USA \\ e Space Physics Data Facility, Code 612.4, NASA Goddard Space Flight Center, Greenbelt, MD 20771, USA \\ ${ }^{\mathrm{f}}$ Universities Space Research Association, NASA Marshall Space Flight Center, XD 12, Huntsville, AL 35812, USA
}

Received 14 September 2004; revised 25 February 2005

Available online 8 August 2005

\begin{abstract}
Remote observations with the Chandra X-ray Observatory and the XMM-Newton Observatory have shown that the jovian system is a source of X-rays with a rich and complicated structure. The planet's polar auroral zones and its disk are both powerful sources of X-ray emission. Chandra observations revealed X-ray emission from the Io plasma torus and from the Galilean moons Io, Europa, and possibly Ganymede. The emission from the moons is due to bombardment of their surfaces by highly energetic magnetospheric protons, and oxygen and sulfur ions. These ions excite atoms in their surfaces leading to fluorescent X-ray emission lines. These lines are produced against an intense background continuum, including bremsstrahlung radiation from surface interactions of primary magnetospheric and secondary electrons. Although the X-ray emission from the Galilean moons is faint when observed from Earth orbit, an imaging X-ray spectrometer in orbit around one or more of these moons, operating from $200 \mathrm{eV}$ to $8 \mathrm{keV}$ with $150 \mathrm{eV}$ energy resolution, would provide a detailed mapping of the elemental composition in their surfaces. Surface resolution of $40 \mathrm{~m}$ for small features could be achieved in a 100-km orbit around one moon while also remotely imaging surfaces of other moons and Jupiter's upper atmosphere at maximum regional resolutions of hundreds of kilometers. Due to its relatively more benign magnetospheric radiation environment, its intrinsic interest as the largest moon in the Solar System, and its mini-magnetosphere, Ganymede would be the ideal orbital location for long-term observational studies of the jovian system. Here we describe the physical processes leading to X-ray emission from the surfaces of Jupiter's moons and the properties required for the technique of imaging X-ray spectroscopy to map the elemental composition of their surfaces, as well as studies of the X-ray emission from the planet's aurora and disk and from the Io plasma torus.
\end{abstract}

(C) 2005 Elsevier Inc. All rights reserved.

Keywords: Instrumentation; Experimental techniques; Jupiter, magnetosphere; Io; Europa; Ganymede; Callisto; Aurorae; Abundances; Impact processes

\section{Introduction}

Besides our Sun, the Jupiter system is the most interesting, powerful and diverse source of X-rays within the Solar System. Observations have identified four distinct sources

\footnotetext{
* Corresponding author. Fax: +1 2569617522.

E-mail address: ron.elsner@nasa.gov (R.F. Elsner).
}

of X-ray emission: (1) the high-latitude auroral zones on Jupiter (Metzger et al., 1983; Waite et al., 1994; Gladstone et al., 2002); (2) the disk of Jupiter (Waite et al., 1997; Maurellis et al., 2000); (3) the Io plasma torus (IPT, Elsner et al., 2002); and (4) the Galilean satellites Io and Europa, with an indication of emission from Ganymede (Elsner et al., 2002). Although these X-ray emissions are faint when observed from Earth orbit, an imaging X-ray spectrometer 
on board a spacecraft moving within the jovian system (e.g., in orbit about the planet or the individual Galilean satellites) would provide a superb capability for unprecedented X-ray studies of Jupiter's auroral and disk X-ray emissions, the IPT X-ray emission, and through measurement of fluorescent line intensities, perhaps most importantly of all, the elemental composition of the surfaces of Jupiter's icy moons and of Io. Such studies can also provide a probe of magnetospheric plasma interactions with Jupiter's upper atmosphere and the Galilean moons.

All of the X-ray phenomena that result from magnetospheric processes could be studied with a Jupiter-based observing platform, which would have unprecedented temporal, spatial, and spectral coverage. Tremendous progress could be made in understanding the surface composition of the icy satellites, auroral processes, the global magnetospheric electrical circuits, and perhaps even the interior structure of Jupiter. In this paper we review what is presently known about the magnetospheric interactions leading to the observed X-ray emissions, and discuss what can be learned from a sensitive imaging X-ray spectrometer within the jovian system.

\section{X-ray emission and scattering processes}

A number of interactions among electrons, protons, ions, neutral atoms and electromagnetic fields lead to the production of X-rays (see the books by Tucker, 1975; Rybicki and Lightman, 1979). One of the simplest is the interaction between an electron and proton or ion leading to the emission of a photon. The electron begins and ends unbound to the heavy positively charged particle. This process is called bremsstrahlung and leads to broadband continuum emission. For sufficiently energetic electrons, the spectrum can peak in the X-ray band or at higher frequencies. The spiraling of a nonrelativistic electron in a magnetic field leads to cyclotron radiation at discrete harmonics of the cyclotron frequency. At higher electron energies the harmonic line emission spreads out forming a continuum; at relativistic energies this is referred to as synchrotron radiation. The shape of the X-ray spectrum can be altered by Compton scattering in which an X-ray scatters off an electron either losing energy, if the electron energy is less than the photon energy, or gaining energy, if the electron energy is greater than the photon energy. Recombination in which an ion captures a free electron produces a continuum spectrum with a sharp low energy cutoff at the binding energy of the atomic level involved. Recombination is the inverse process to photo-ionization in which a photon is absorbed by an atom or ion, kicking out an electron and increasing the charge state by one. If the ejected electron comes from an inner shell, one of the outer electrons will drop to the lower level causing either the ejection of a second (Auger) electron from an outer level or the emission of a fluorescent photon with energy equal to the energy difference be- tween the two levels. When the ejected electron comes from the innermost energy level (the $\mathrm{K}$ shell), then for elements from carbon through iron, the energy of the fluorescent photon will lie in the X-ray band between $277 \mathrm{eV}$ (for carbon) to $6.4 \mathrm{keV}$ (for iron). Fluorescent emission can also occur when the outer electron drops into a level above the $\mathrm{K}$ shell (say the L shell), however for most of the elements of interest the energies of such transitions lie below the X-ray band. The L shell fluorescent lines for iron are at 705 and $718 \mathrm{eV}$, which is within the X-ray band of interest to us; however, the $\mathrm{L}$ shell fluorescent yield is much less than for the $\mathrm{K}$ shell.

Also important in the present context are interactions between ions and neutral atoms or other ions. In the process known as collisional ionization, an interaction between an energetic ion or electron with a neutral atom leads to the ejection of an electron from the atom. If the ejected electron is from the outermost energy level, as is most likely, a free electron will eventually recombine with the newly formed ion leading to the emission of a low energy photon typical of recombination. If the electron is from an inner energy level, it is likely that an electron from an outer level will fall to the inner level with the emission of a fluorescent photon, and eventually recombination to the outer lever will also occur. If the inner energy level is the $\mathrm{K}$ shell, then for carbon through iron, as above, the energy of the fluorescent line emission will fall in the band $277 \mathrm{eV}$ to $6.4 \mathrm{keV}$. Collisional ionization can also occur between two ions, but the line energies will be different because the energy levels in the ion are somewhat different, depending on the degree of ionization, from those in the neutral atom. In the charge exchange process (cf. Cravens, 1997, 2002), the energetic ion captures an electron from the neutral atom, reducing the charge state of the incident ion by one but leaving it in an excited state. Subsequently this excited product ion de-excites and emits a fluorescent photon, or even two if the de-excitation occurs in two stages. If the excited product ion has a vacancy in the lowest energy level (K shell) then X-ray line emission can occur as already discussed.

All of these processes occur in the jovian environment. Which ones dominate depends on the relative crosssections for the processes, and the particle energies, densities, and electromagnetic field strengths of the particular environment. Jupiter's auroral X-ray emission is attributed to charge-exchange between energetic ions and neutral atoms high in the polar atmosphere (cf. Bhardwaj and Gladstone, 2000, and references therein). X-ray emission from the Galilean moons may result from the energetic ions incident on their surfaces ionizing and exciting neutral surface atoms leading to fluorescent K-shell line emission (Elsner et al., 2002). A detailed understanding of the $X$-ray emissions from the jovian system requires detailed modeling of the complex interactions to be compared with high-quality X-ray spectra. We are presently engaging in construction of a detailed model for the X-ray emission from Jupiter's icy moons. 


\section{Jupiter's X-ray aurora}

High spatial resolution $(<1$ arcsec $)$ observations of Jupiter with the Chandra X-ray Observatory (CXO) HRC-I $X$-ray camera on 18 December 2000 (Gladstone et al., 2002) located most of the auroral X-rays within small high-latitude regions that in the north were confined in longitude between $160^{\circ}$ and $180^{\circ} \mathrm{S}_{\mathrm{III}}$ and latitude $60^{\circ}$ and $70^{\circ}$. Thus the auroral $\mathrm{X}$-ray emission region was strongly correlated with Jupiter's magnetic field and mapped along magnetic field lines to distances $>30$ jovian radii from the planet. CXO observations on 24-26 February 2003 obtained a better view of the southern auroral emission and found it to be more extended in longitude than in the north, but still confined to a region that mapped along field lines far out into the magnetosphere (Elsner et al., 2005). Thus the X-ray auroral emission regions lie well inside the UV auroral oval, with important implications for the current systems accelerating the incoming ion beam (Cravens et al., 2003; Bunce et al., 2004; Elsner et al., 2005). Typically the auroral regions emit $\sim 0.5-$ $1.0 \mathrm{GW}$ in soft X-rays.

The $\mathrm{X}$-ray emission from the auroral region is variable. Short (seconds to minutes) UV flares (Waite et al., 2001) have been observed in one case to be accompanied by an X-ray flare (Elsner et al., 2005), with the location of the X-ray flare apparently slightly displaced from that of the UV flare (Elsner et al., 2005). This displacement could have important implications for understanding magnetospheric current systems and interactions at the magnetopause (Bunce et al., 2004). More examples of such flares, with better statistics obtainable only with an instrument close or within the jovian system, are needed to fully understand this behavior.

The 18 December 2000 CXO observation unexpectedly found a statistically significant $\sim 40$ min quasi-periodic oscillation in the $\mathrm{X}$-ray emission from the northern auroral zone (Gladstone et al., 2002). The viewing geometry for the southern auroral region was not favorable for this observation, and the existence of such oscillations there could not be established with confidence. The capacity of the jovian system for surprises in the X-ray band displayed itself again when the $\sim 40$ min oscillations were not found in the X-ray emission from either auroral region during the 24-26 February 2003 CXO observations, although the emission did show variability on timescales from 10 to $100 \mathrm{~min}$ (Elsner et al., 2005). Ulysses has also observed forty minute oscillations, correlated with quasi-periodic radio bursts from Jupiter, with in situ measurements of $\sim 16 \mathrm{MeV}$ relativistic electrons and energetic ions in February 1992 (McKibben et al., 1993; MacDowall et al., 1993; Karanikola et al., 2004). At this time Ulysses was at high jovimagnetic latitudes on field lines likely connected to the southern jovian polar cap. However, at the time of the 2003 CXO observations, the Ulysses radio data did not show strong 40 min quasi-periodic oscillations, although variability on timescales similar to that in the X-ray data was present.

Recent XMM-Newton (Branduardi-Raymont et al., 2004) and CXO (Elsner et al., 2005) observations have established beyond doubt that the relatively bright $\mathrm{X}$-ray emission from Jupiter's polar regions is line emission, rather than a continuum, with the mechanism almost certainly being charge exchange between energetic highly-stripped heavy ions and neutral atoms in Jupiter's upper atmosphere. The CXO X-ray spectrum for the northern auroral region is shown in Fig. 1. There is strong evidence that one major constituent of the incoming ion stream is highly ionized oxygen (OVII and OVIII), responsible for X-ray line emission in the 500-900 eV energy band. There must be at least one other major constituent to account for the line emission at $400 \mathrm{eV}$ and below. Two candidates are L-shell

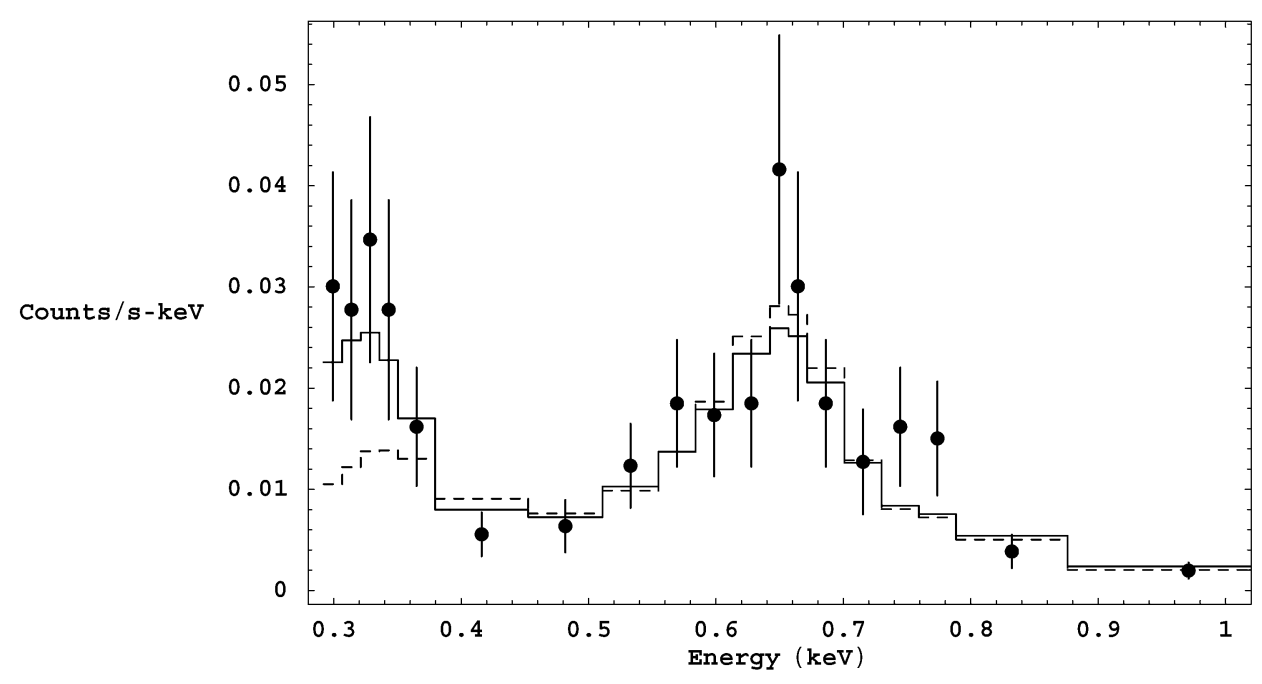

Fig. 1. CXO spectrum for the northern auroral zone from February 2003. The solid line represents a model fit assuming collisional equilibrium and the presence of only oxygen and sulfur ions, while the dashed line represents a model fit for only oxygen and carbon. OVII and OVIII transitions account for the emission in the energy band around $650 \mathrm{eV}$. The low energy emission is due either to SX, SXI, and SXIV transitions or CV and CVI transitions. The oxygen-sulfur case results in a better fit. See Elsner et al. (2005) for a fuller discussion. 
transitions in highly ionized sulfur and K-shell transitions in highly ionized carbon. The identification of sulfur as the second main constituent would favor a magnetospheric origin for the incoming stream of ions, while carbon would favor a solar wind origin. Under the model assumptions made for line positions and intensities in the spectral analyses, CXO data seem to favor sulfur while XMM-Newton data, taken two months later in April 2003, seem to favor carbon. Jupiter's near infrared/optical brightness requires special setups for both observatories so that the relatively small number of low energy photons is a factor limiting the sensitivity of the spectral analysis. However, it is clear that quality X-ray spectra as a function of both space and time can provide a direct probe of magnetospheric flows and possibly even reconnection episodes at the magnetopause.

There is an interesting potential connection here to the yet-unknown source of $\mathrm{CO}_{2}$ on the surface (Hibbitts et al., 2000) and in the atmosphere (Carlson, 1999) of Callisto. Johnson et al. (2004) considered solar wind carbon irradiation as one possible source of the surface $\mathrm{CO}_{2}$, and surface sputtering could generate the atmospheric component. In order for the carbon ions to primarily impact the trailing hemisphere, the ion gyroradii in the weak $\sim 10 \mathrm{nT}$ magnetic field must be much less than the moon radius of $2400 \mathrm{~km}$, requiring energies much less than a few $\mathrm{keV}$ for fully stripped heavy ions (Cooper et al., 2001), but the full magnetospheric corotation energy available to any carbon ion at Callisto's orbit is much higher at about $6 \mathrm{keV}$. This problem is resolved if the carbon ions are of solar wind origin and fully charged $\left(\mathrm{C}^{6+}\right)$, so that typical energies of these ions impacting the trailing hemisphere can be near or even much greater than the corotation energy. Mapping of X-ray emission from charge exchange reactions of carbon and other ions impacting onto the surfaces of Callisto and other Galilean moons could then give limits on the ion charge states and origins independent of direct in situ measurements not yet available or reported.

Problems with the sulfur source for auroral emissions are that sulfur and other ions from the Io plasma torus likely have low charge states (Hamilton et al., 1981; Geiss et al., 1992), and UV absorption observations for Callisto (e.g., Calvin and Clark, 1993; Calvin et al., 1995) suggest sulfur mostly on the leading hemisphere, suggestive of a nonmagnetospheric source such as meteoritic bombardment. Thus the observed auroral emissions, and the sources of ion irradiation for Callisto, could be from solar wind oxygen and carbon ions with little input from iogenic sulfur, consistent with expected penetration of solar wind ions into Jupiter's outer magnetosphere.

Prior to the high-spatial-resolution CXO observations of Jupiter, the expectation was that the auroral X-ray emission lay along field lines threaded through the Io plasma torus, and hence would be concentrated just northward of the foot of the Io flux tube. Since the feet of satellite flux tubes may well be sites of energetic particle precipitation, they may also be sources of X-ray emission, just not as strong as the observed aurora.

\section{X-ray emission from the planetary disk}

Even with CXO's superb angular resolution, corresponding to $\sim 5 \%$ of Jupiter's equatorial radius at $4.5 \mathrm{AU}$ (typical Earth-Jupiter separation during the X-ray observations), away from the polar regions Jupiter's X-ray emission appears to be a spatially featureless disk illuminated by solar X-rays (Gladstone et al., 2002; Elsner et al., 2005). Furthermore, the X-ray spectrum (shown in Fig. 2) appears consistent with elastic scattering of solar X-rays by atmospheric neutrals and carbon K-shell fluorescent X-ray emission from methane molecules located below the jovian homopause (Maurellis et al., 2000). The connection of Jupiter's disk

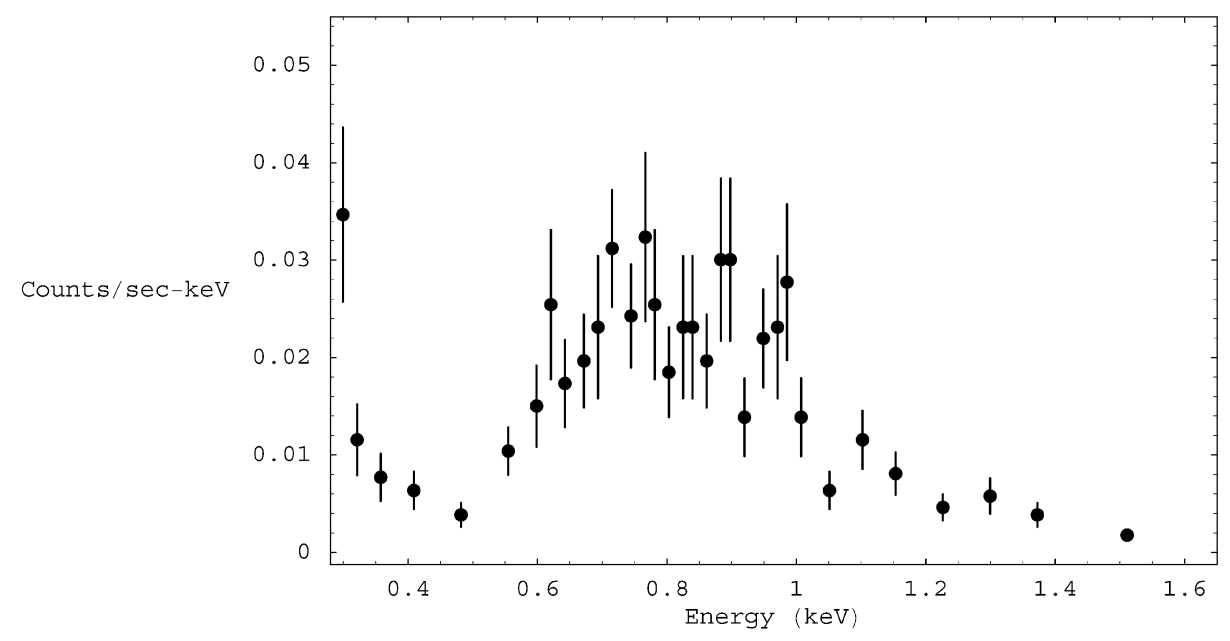

Fig. 2. CXO spectrum for the planetary disk away from the northern and southern auroral zones. The spectrum extends to higher energies than the auroral spectrum and its shape is different. This emission is presumably due to scattering and reprocessing of incident solar X-rays (Maurellis et al., 2000). Cravens et al. (2005, in preparation) are engaged in more extensive modeling as part of the detailed analysis of this spectrum. 
$\mathrm{X}$-ray emission to solar X-ray emission is strengthened by the temporal correlations reported by Bhardwaj et al. (2005) when light travel time effects are taken into account. However, more detailed modeling and comparison with data of high statistical quality are still needed, especially to look for possible deviations from this simple picture due, for example, to precipitation of energetic sulfur or oxygen ions into the atmosphere from the radiation belts (Gehrels and Stone, 1983; Waite et al., 1997; Gladstone et al., 1998) and possible correlations with surface magnetic field strength. Jupiter's disk typically emits $\sim 2 \mathrm{GW}$ in soft X-rays, but with a somewhat harder spectrum than the aurora X-rays.

\section{X-ray emission from the IPT}

CXO observations on 25-26 November 1999 and 18 December 2000 detected a faint diffuse source of soft X-rays from the region of the IPT (Elsner et al., 2002). The 2000 CXO image, obtained with the HRC-I camera, exhibited a dawn-dusk asymmetry similar to that seen in the EUV (Hall et al., 1994; Gladstone and Hall, 1998). The 1999 CXO image, taken with the ACIS-S camera, showed more complicated structure not readily interpreted (the 1999 ACIS-S data from the planet were rendered useless by contamination from its bright optical disk (Elsner et al., 2002); the 2003 ACIS-S observations were specifically designed to eliminate this problem (Elsner et al., 2005)). Fig. 3 shows the background-subtracted CXO/ACIS-S IPT spectrum for 2526 November 1999. This spectrum shows evidence for line emission centered on $574 \mathrm{eV}$ (very near a strong OVII line), together with a very steep continuum spectrum at the softest X-ray energies. There could be contributions from other charge states, since current plasma torus models (Bagenal et al., 1992; Bagenal, 1994) consist mostly of ions with low charge states, consistent with photoionization and ion- neutral charge exchange in a low-density plasma and neutral gas environment. Keeping in mind that the calibration of the ACIS energy response is most uncertain at these very low energies, the corresponding power emitted by the IPT was $\sim 0.12 \mathrm{GW}$. Elsner et al. (2002) were unable to provide a convincing physical mechanism for the observed IPT X-ray emissions, although they noted that bremsstrahlung from nonthermal electrons might account for a significant fraction of the continuum X-rays. The 2003 CXO observations also observed X-ray emission from the IPT, although at a fainter level than in 1999 or 2000 . The morphology exhibited the familiar dawn-dusk asymmetry. These IPT data are not yet fully analyzed and will be reported on in a future paper.

Knowledge of the spatial structure in X-rays and X-ray spectra of the IPT are limited by its faintness and would be vastly improved by observations from a spacecraft approaching Jupiter. A region $10 R_{\mathrm{J}}$ in radius fits within, for example, a $6^{\circ}$ field of view at distances of $200 R_{\mathrm{J}}$ or greater. On approach to the jovian system an imaging X-ray spectrometer would be able to continuously observe the X-ray emission from the IPT, and accurately measure its spatial structure in X-rays and its X-ray spectrum. Thus far $\mathrm{Na}, \mathrm{Cl}, \mathrm{S}$, and $\mathrm{O}$ ions, and possibly protons, have been seen in the IPT, but there must be other species present as well, especially in view of the constant replenishment of material from Io. A $10-R_{\mathrm{J}}$ observational radius for the IPT also encompasses the orbit of Europa, where neutral $\mathrm{Na}$ (Brown and Hill, 1996) and $\mathrm{K}$ (Brown, 2001) have been detected, and the $\mathrm{Na}$ may predominantly originate from sputtering off of that icy moon's surface (Johnson, 2000; Leblanc et al., 2002). Other potential Europa contributions to the outer IPT via surface sputtering include $\mathrm{C}$ from observed surface $\mathrm{CO}_{2}$ (Smythe et al., 1998; Carlson, 2001), Mg from magnesium sulfate hydrates suggested to be consistent with Galileo near-infrared spectroscopy (McCord et al., 1998a, 1999), and the neutral

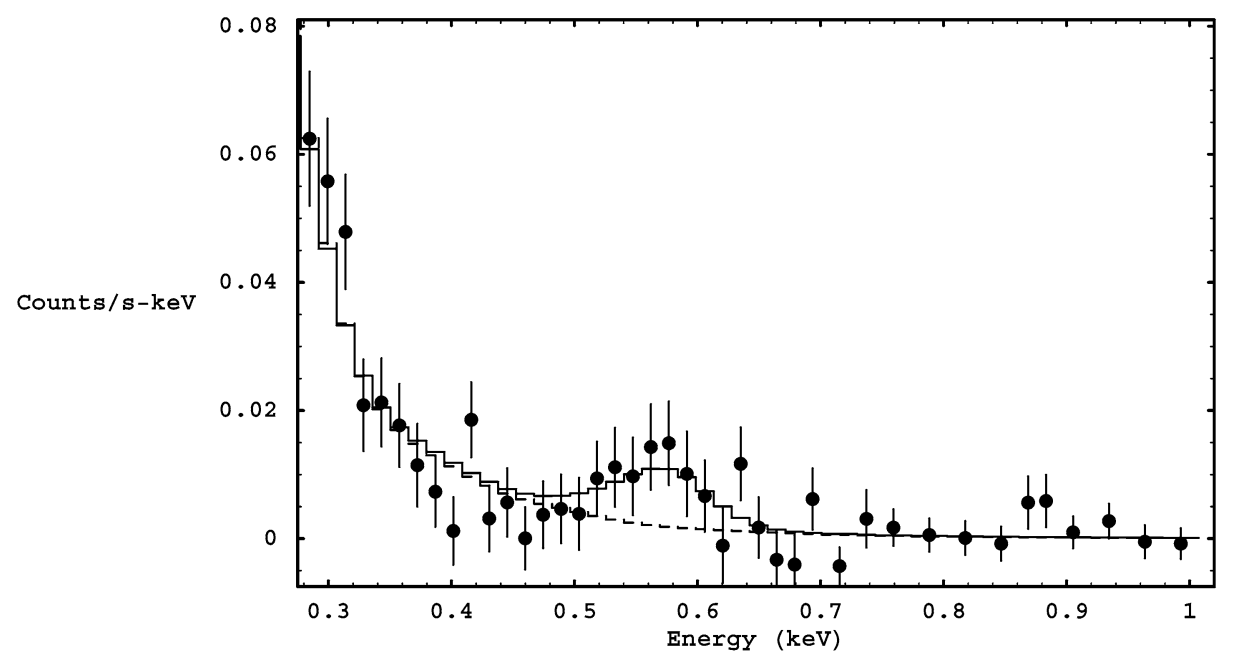

Fig. 3. CXO spectrum for the Io plasma torus from November 1999. The solid line represents a model fit for the sum of a power-law spectrum and a Gaussian line, while the dotted line presents just a pure power-law spectrum. The line is consistent with K-shell fluorescent line emission from oxygen ions. See Elsner et al. (2002) for a fuller discussion. 
Europa torus composed of water ice and other dissociation products sputtered off the moon's surface (Mauk et al., 2003; Shematovich et al., 2005).

\section{X-ray emission from the Galilean satellites}

The 1999 and 2000 CXO observations also discovered $\mathrm{X}$-ray emission from the Galilean satellites (Elsner et al., 2002). These satellites are very faint when observed from Earth orbit and the detections of Io and Europa, while statistically very significant, were based on $\sim 10$ photons each, a tribute to the high spatial resolution and low background of the CXO. The energies of the detected X-ray events ranged between 300 and $1890 \mathrm{eV}$ and appeared to show a clustering between 500 and $700 \mathrm{eV}$, suggestive of oxygen K-shell fluorescent emission. The estimated power of the $\mathrm{X}$-ray emission was $2 \mathrm{MW}$ for Io and $3 \mathrm{MW}$ for Europa. There were also indications of X-ray emission from Ganymede. X-ray emission from Callisto seems likely at levels not too far below the CXO sensitivity limit, since the magnetospheric heavy ion fluxes (Cooper et al., 2001) are an order of magnitude lower than at Ganymede and Europa, respectively. No Galilean satellite was detected during the 24-26 February 2003 CXO observations. However, both Jupiter's disk and the IPT were about $1 / 2$ as bright as in the earlier observations; reduction in the emission from the moons by a similar factor is likely the cause of the failure to detect them in the new observations.

The emissions from Europa are best explained by energetic $\mathrm{H}, \mathrm{S}$, and $\mathrm{O}$ ion bombardment of the icy surface and subsequent fluorescent emission from the deposition of energetic particle energy in the top $10 \mu \mathrm{m}$ of the surface, which is optically thin to outgoing $\mathrm{X}$-ray emission. For a stopping range of $10 \mu \mathrm{m}$ in unit density $\mathrm{H}_{2} \mathrm{O}$ the corresponding ion energies for $\mathrm{H}, \mathrm{S}$, and $\mathrm{O}$ are 540,18 , and $10 \mathrm{MeV}$, respectively, from the SRIM (Stopping and Range of Ions in Matter) model of Ziegler et al. (1985) now updated for 2003 (see http://www.srim.org/index.htm). Higher energy ions passing through this layer also deposit energy in decreasing amounts with increasing energy. For electrons the stopping energy in this layer is $20 \mathrm{keV}$, and most of the electron energy flux is at much higher energy (Cooper et al., 2001), so the minimumionizing magnetospheric electrons deposit relatively little energy for X-ray production in this layer but do contribute to the scattered continuum from deposition at greater depths. However, much of the low-energy electron flux may be deflected away from the surface (Saur et al., 1998) by electric and magnetic fields associated with ionospheric currents.

Although the X-ray emissions are weak as viewed from Earth they will be very bright to an X-ray instrument in orbit about the moons. High spectral and spatial resolution measurements with such an instrument can be used to determine the elemental composition of the surface material including the minor constituents present in the surface layer. Furthermore, in conjunction with on-orbit mass spectrometry of sputtered ions, atoms, and molecules, X-ray observations can be used to determine element, molecular, and isotopic composition of surface material—an ideal "secondary mass spectrometer in the sky" enabled by the unique location of the satellites within the pervasive ion gun of Jupiter's outer radiation belts.

The incident flux on the moons is made up mostly of energetic H, O, and S ions (Paranicas et al., 1999, 2002a; Cooper et al., 2001) with a lesser amount of He (Mauk et al., 2004). Although Europa has an ionosphere that partly deflects lowenergy $(<10 \mathrm{keV})$ magnetospheric ions (Saur et al., 1998; Paranicas et al., 2002b), more-energetic ( $>10 \mathrm{keV})$ ions preferentially reach its surface and produce a large flux of X-rays (Johansson et al., 1995). Since higher charge states lose their energy more quickly, the more penetrating $\mathrm{MeV}$ ions, which rapidly lose their remaining atomic electrons on initial entry into the ice before reaching stopping range depths of microns to centimeters (increasing with energy) become particularly important for X-ray production. The relatively larger gyroradii for these ions means that the effective cross-section of Europa for impact of ions producing X-ray fluorescent lines can be larger than its physical size (Pospieszalska and Johnson, 1989), although the magnetosphere-moon interaction is complex and some ions within the gyroradius distance do pass downstream without impact. Magnetohydrodynamic (MHD) simulations have been done on the plasma-moon interaction but the needed hybrid modeling for energetic ion interactions, as discussed by Cooper et al. (2001), is only now in progress. Initial calculations of Elsner et al. (2002) showed that estimates of the expected X-ray flux due to $\mathrm{K}$ shell fluorescence of the oxygen in the ice on Europa, using measurements of the expected $\mathrm{H}, \mathrm{O}$, and $\mathrm{S}$ flux at Europa and taking into account absorption in the surface layers, were within a factor of three of the measured X-ray flux. They noted that $\mathrm{O} K_{\alpha} \mathrm{X}$-rays at $525 \mathrm{eV}$ have an optical depth in ice of about $10 \mu \mathrm{m}$; according to irradiation models (Paranicas et al., 2001; Cooper et al., 2001), ion energy losses dominate those from electrons in this layer. Improved in situ X-ray measurements would be able to greatly extend this work to map the elemental abundances over the surface of Europa, and extend these measurements to Ganymede and Callisto as well.

An essential question is whether the strength of the continuum, ultimately mostly due to deceleration of the incident electrons, plus the already high instrumental background, due mostly to electron interactions in the instrument housing or to ion and electron fluxes entering the aperture facing the moon, is low enough to permit sufficiently precise comparisons of measured line intensities with the models. As always in spectroscopy, the better the energy resolution of the detector, the easier it becomes to detect lines against a strong continuum and the easier it is to separate lines from different charge states. The energy of the electrons is mostly deposited in deeper layers from which X-rays cannot freely escape. The intensity and spectral shape of the continuum will depend on the radiative transfer from those deep lay- 
ers. The portion of the deposited energy that is turned into heat will ultimately escape as photons with energies far below the X-ray band of interest. Another essential question, in the case of the incident $\mathrm{O}$ and $\mathrm{S}$ ions, is what fraction of the $\mathrm{O}$ and $\mathrm{S} \mathrm{K}$-shell fluorescent line emission is due the incident $\mathrm{O}$ and $\mathrm{S}$ ions rather than the neutral $\mathrm{O}$ and $\mathrm{S}$ atoms already in the surface. As stated above, the penetrating ions will rapidly lose their remaining atomic electrons before stopping. Their energies are so much higher than the binding energy of $\mathrm{K}$ shell electrons for neutral atoms that each ion will interact with a great many surface atoms before stopping. A large number of these interactions will result either in the escape of outer shell electrons or their transfer to the energetic ions possibly resulting in fluorescent line emission, and some but not all of this charge exchange line emission will be in the $\mathrm{K}$ shell lines. A fraction of the emission due to charge transfer to the incident ions will also fall outside the X-ray band. Some of the interactions with incident ions will result in $\mathrm{K}$ shell vacancies in the neutral atoms in the surface. The balance between these competing possibilities is the result of a complex cascade of processes, and these questions require a quantitative treatment beyond the scope of this paper. We are presently engaged in modeling the interaction of the incident ions and electrons with putative surface compositions in order to answer such questions, to predict the emitted X-ray spectra and to more adequately quantify our assertions.

The surface compositions of the icy moons are expected to be rich in interesting chemical species (e.g., Kargel et al., 2000; Fanale et al., 2001; Zolotov and Shock, 2001; Pierazzo and Chyba, 2002; Johnson et al., 2003, 2004; McKinnon and Zolensky, 2003) and their surfaces (McCord et al., 1998a, 1998b; Carlson et al., 1999, 2005) and associated atmospheres (McGrath et al., 2004) display significant spatial variability. Observed time variations in UV absorption (Lane and Domingue, 1997; Domingue and Lane, 1998; Domingue et al., 1998; Domingue and Hendrix, 2005) also indicate that related surface composition for Europa, Ganymede, and Callisto varies with time in irradiated upper layers, probably mostly due to changes in magnetospheric activity but potentially also due to transient emergence of material from internal sources. The recent identification of the products of radiolysis of water ice $\left(\mathrm{O}_{2}, \mathrm{O}_{3}, \mathrm{H}_{2} \mathrm{O}_{2}\right)$ and the identification of species formed from $\mathrm{S}$ and $\mathrm{C}$ in ice have been exciting (e.g., Johnson et al., 2004). Far more speculative but intriguing is the (admittedly remote) possibility (Dalton et al., 2003) that remnants of biological materials may have contributed to the observed infrared spectra.

Measurements of trace species are needed to distinguish between models for surface and interior geology, atmospheric evolution, presence of subsurface oceans strongly suggested by Galileo magnetometer results, and astrobiological potential at each moon. The only clearly identified trace species seen are $\mathrm{Na}$ and $\mathrm{K}$ as detected remotely in the extended neutral atmosphere of Europa, but not at the surface and not at all at Ganymede and Callisto. To understand sur- face and interior composition for all the icy Galilean moons, we need global maps, correlated at good spatial resolution to geologic features, of the known or inferred elements such as $\mathrm{C}, \mathrm{O}, \mathrm{S}, \mathrm{Na}, \mathrm{Mg}, \mathrm{K}, \mathrm{Cl}$, based on currently available observations of the IPT and the Galilean moons, and other species (N, Ca, P, Fe, etc.) yet to be identified. Although $\mathrm{X}$-ray fluorescence provides no information on molecular species such as the much-debated sulfate hydrates, comprehensive elemental measurements will allow some modeling of molecular composition from abundances relative to the most common element, oxygen.

Most of these considerations also apply to orbiting observations of the very different surface of Io. The major source of change in surface composition on this moon is volcanic eruptions, but there are some indications in polar regions of magnetospheric irradiation effects (Johnson et al., 2004). In view of the very intense radiation environment at Io, which affects both the X-ray instrument background and spacecraft operational lifetime, more remote observations may be preferable. Io orbits Jupiter at a distance of about $6 R_{\mathrm{J}}$, so that its entire orbit fits within a $6^{\circ}$ field of view at distances of $120 R_{\mathrm{J}}$ or greater. Thus on approach of a host spacecraft to the jovian system an imaging X-ray spectrometer would be able to continuously observe the X-ray emission from Io, except when it lies behind the planet. Additional, higherresolution observations could take place when the spacecraft is transiting between the icy moons. In this way one would obtain abundances for Io integrated over its surface, and time variations could be monitored during years of spacecraft orbital operations in the jovian system.

\section{X-ray mapping of icy moon elements}

To illustrate the science that can be obtained from an $\mathrm{X}$-ray instrument within the jovian system we briefly describe some properties of an imaging X-ray spectrometer designed to survey elemental composition of the icy moons in the Jupiter system and carried by a future high-capacity (power, telemetry, weight) mission. The instrument would be designed to map, through measurement of fluorescent $\mathrm{X}$-rays induced by charge particle bombardment, the elemental abundances of the Galilean moons while in orbit around each. If, as might be the case for Io or possibly Europa in a near-term mission, the radiation environment precludes orbiting the moon for useful periods of time, values for the abundances integrated over the surface would be obtained from a greater distance. Remote X-ray imaging of these moons at regional resolution and of Jupiter could be achieved in orbit around Ganymede in its less intense radiation environment, while also exhaustively surveying surface composition of this largest moon in the Solar System and its polar auroral emissions arising from jovian magnetospheric interactions with the intrinsic dipole magnetic field and mini-magnetosphere (Kivelson et al., 2004). 
The instrument would also provide spatially, spectrally, and temporally resolved maps of Jupiter's X-ray emission, especially from the auroral zones, and of the IPT. This would be accomplished during the approach to Jupiter, during transits between moons, or in carefully planned flybys of interesting regions. Thus such an instrument could provide valuable observations of the jovian system even without close flybys or orbital operations at the Galilean moons.

\subsection{General description}

Operating within the harsh radiation environment within the Jupiter system is a serious challenge for any instrument. The high background rate severely limits the sensitivity of any X-ray detector and in addition the extremely high counting rate anticipated and the possibility of radiation-induced damage place stringent demands on the design.

To overcome the high background and obtain sensitive measurements, and for stand-off imaging of Jupiter, Io, and the IPT, focusing X-ray optics must be used. These permit collection of significant source fluxes, through the use of large mirror areas, but focus these to a small focal spot so that the detector-area-dependent background becomes manageable.

An angular resolution of about $1 \frac{1}{2}$ arcmin, a fairly modest performance for current $\mathrm{X}$-ray optics, at an altitude of $\sim 100 \mathrm{~km}$ implies a surface resolution of around $40 \mathrm{~m}$. Maximum surface resolution for remote imaging of Europa and Io from Ganymede at minimum separation distances $\left(5-9 R_{\mathrm{J}}\right)$ would be 140 and $260 \mathrm{~km}$, respectively, sufficient for compositional mapping of distinct geologic regions. As X-ray mirrors have very shallow reflection, or graze, angles, $a \sim 6^{\circ}$ field of view could in principle be obtained by utilizing a $3 \times 3$ array of telescopes, each with a $\sim 2^{\circ}$ field of view and offset from each other by $2^{\circ}$. Because of the shallow graze angle, individual mirrors have very small collecting areas, and so to obtain significant source fluxes multiple shells are typically nested within a single telescope, as has been done with X-ray astronomy missions including CXO and XMMNewton. A sufficiently capable optical system might use 30 nested mirror shells per telescope, each spaced by $4 \mathrm{~mm}$ and covering a diameter range from 104 to $336 \mathrm{~mm}$. The focal length of each telescope would be $1.2 \mathrm{~m}$, and, for $1 \frac{1}{2}$ arcmin angular resolution, an imaging X-ray detector with $\sim \frac{1}{2} \mathrm{~mm}$ spatial resolution is then required at the focus of each.

The fluorescent X-ray lines from light surface elements are at relatively low energies and need to be measured with good energy resolution to separate them from each other and from the pervasive background. Ideally, to ensure full line flux collection for the lowest line energy (carbon at $0.27 \mathrm{keV}$ ), the detector threshold should be below $0.2 \mathrm{keV}$ and the detector energy resolution should be below $150 \mathrm{eV}$ FWHM. These are very challenging requirements. Further, for any focal plane instrument on a mission within Jupiter's magnetosphere, consideration must be made for the harsh environment in which it must operate. First and foremost, the imaging X-ray detector must be able to survive the extremely high radiation dose that it will receive, particularly during those periods the spacecraft spends in the inner magnetosphere (for example, in orbit around Europa or Io). Assuming a few months around the three icy Galilean satellites (Europa, Ganymede, and Callisto), crude estimates, using ion and electron data from Cooper et al. (2001) and Paranicas et al. (2002a, 2002b), indicate that with significant shielding surrounding all but the entrance beam from the focusing optics, we could expect around $0.25 \mathrm{Mrad}$ of accumulated dose.

This accumulated dose rules out traditional X-ray CCD imagers, which normally would be the focal plane detector of choice for low-energy, high-resolution imaging. X-ray CCDs can be severely degraded by all forms of ionizing radiation (e.g., Miyata et al., 2003). Furthermore, depending on the shielding employed and its efficiency, one can expect raw detector count rates anywhere from $\sim 10^{4}$ to $\geqslant 10^{6}$ counts $\mathrm{cm}^{-2}-\mathrm{s}^{-1}$. This too is beyond the capability of CCD imagers.

There are two possible types of detectors that may satisfy the requirements outlined above: (1) pixellated silicon PIN diode arrays (Krieger et al., 2001); and (2) pixellated silicon drift detectors (Struder, 2000). These types of detectors afford direct readout of individual pixels through intimately bonded electronics, with no charge clocking or large distances for charge packets to travel, thus permitting high speed operation and high radiation tolerance. In addition, with no coupling wires and small pixels, very low noise levels are possible. Both types of detectors have been demonstrated in array form, coupled to custom built readout electronics, called Application-Specific Integrated Circuits (ASICS). These ASICS contain hundreds or thousands of discrete readout channels on a single integrated circuit. When intimately connected to the silicon detector, usually through bump bonding, they can provide the lowest noise, highest speed readouts possible. Both detector types have demonstrated performance approaching that needed for the focal plane detectors.

The resources needed by this imaging $\mathrm{X}$-ray spectrometer deserve mention. Because of the background environment the detectors will be required to run at raw rates of at least ten thousand counts per $\mathrm{cm}^{2} \mathrm{~s}$ even with substantial shielding, and possibly 10-100 times this. Even with significant on-board rejection, one can still anticipate net data rates of a few thousand per $\mathrm{cm}^{2} \mathrm{~s}$. Assuming $\sim 100 \mathrm{~cm}^{2}$ of total detector area and 50 bits per event, this requires a telemetry rate of at $15 \mathrm{Mb} \mathrm{s}^{-1}$. For high-speed operation, detector electronic considerations indicate that one would need around $8 \mathrm{~mW}$ per channel of front-end electronics power consumption. For the full focal plane, this amounts to $\sim 360 \mathrm{~W}$, with an additional $\sim 700 \mathrm{~W}$ for detector cooling to around $-30{ }^{\circ} \mathrm{C}$. With processing electronics the total power burden comes to around $1.5 \mathrm{~kW}$. These resources, while large when compared to past missions, could, for example, be met by the 
NEPP technologies under development by NASA's Project Prometheus. Although not discussed here, excellent science could also be done by a smaller instrument to be flown on a more conventional mission.

\subsection{Instrument sensitivity}

In 1999 and 2000 the Chandra X-ray Observatory measured a power of $3 \mathrm{MW}$ from Europa, with photon energies mostly consistent with the oxygen $K_{\alpha}$ fluorescent line. The number of photons detected was $\sim 10$, with essentially no background. For isotropic emission uniformly distributed over the surface, this implies an incident flux at a $100 \mathrm{~km}$ altitude of 2.6 photons $\mathrm{s}^{-1} \mathrm{~cm}^{-2} \mathrm{deg}^{-2}$.

The detector background rate on the spacecraft, against which measurements must be made, depends critically on the amount and efficiency of shielding employed and also on-board rejection techniques. While it is easiest to shield the detectors from relatively low energy incident electromagnetic radiation and particles, energetic ions, electrons, and photons interacting with the material in the shield produce a cascade of particles and photons, together with flux entering the aperture, that make up the actual detector background. To estimate the anticipated background in orbit about Europa, we used measured energetic particle flux data at Europa (Cooper et al., 2001; Paranicas et al., 1999, 2001, 2002a). Then, simple but conservative estimates, based on our experience comparing the results of Monte-Carlo simulations with actual satellite and balloon-borne X-ray detector background data, indicate a $0.2-6.0 \mathrm{keV}$ net background rate of $2 \times 10^{4}$ counts s $\mathrm{s}^{-1} \mathrm{~cm}^{-2}$ for medium shielding (2-3 inches) and $2 \times 10^{3}$ counts s $\mathrm{sm}^{-1}$ for heavy shielding $(\sim 8-10$ inches), as a starting point for our sensitivity calculations. These high rates mean that our measurements will be background limited. Detailed modeling of the expected background given the radiation environment at the Galilean moons is being undertaken at the present time. We are im-

Table 1

Europa elemental abundances (in percent) relative to oxygen measurable at $3 \sigma$ with spatial resolution of $420 \times 420 \mathrm{~m}$ from a height of $100 \mathrm{~km}$

\begin{tabular}{|c|c|c|c|c|}
\hline \multirow[t]{2}{*}{ Element } & \multirow{2}{*}{$\begin{array}{l}\text { Energy } \\
(\mathrm{keV})\end{array}$} & \multirow{2}{*}{$\begin{array}{l}\text { Effective } \\
\text { area }\left(\mathrm{cm}^{2}\right)\end{array}$} & \multicolumn{2}{|l|}{ Abundance $(\%)$} \\
\hline & & & Thin shielding & Thick shielding \\
\hline Carbon & 0.277 & 187 & 16 & 5.1 \\
\hline Nitrogen & 0.392 & 184 & 8.7 & 2.8 \\
\hline Fluorine & 0.677 & 181 & 3.5 & 1.1 \\
\hline Neon & 0.849 & 180 & 2.6 & 0.82 \\
\hline Sodium & 1.040 & 179 & 2.0 & 0.65 \\
\hline Magnesium & 1.254 & 172 & 1.6 & 0.52 \\
\hline Aluminum & 1.487 & 161 & 1.3 & 0.42 \\
\hline Silicon & 1.740 & 139 & 1.2 & 0.38 \\
\hline Potassium & 2.014 & 72 & 1.8 & 0.58 \\
\hline Sulfur & 2.308 & 26 & 4.1 & 1.3 \\
\hline Chlorine & 2.622 & 29 & 3.0 & 0.95 \\
\hline Calcium & 3.692 & 20 & 2.6 & 0.83 \\
\hline Iron & 6.404 & 3.4 & 6.2 & 2.0 \\
\hline
\end{tabular}

plementing the GEANT code (http://cern.ch/geant4) to provide the best possible estimates for the expected background. GEANT was originally developed by and for the high energy particle physics community, is undergoing continuous development and enhancement, and has increasingly become a standard tool in the gamma-ray astronomy community and lately the X-ray astronomy community as well. GEANT could also be used to model magnetospheric irradiation effects at the Galilean moon surfaces. On-orbit background determination would of course be an important part of any experiment.

We then calculated, as a function of elemental abundance relative to oxygen, the expected signal to noise ratio per spatial element by taking background over a $300 \mathrm{eV}$ energy bandwidth, the modeled telescope effective area at each line, a detector quantum efficiency of 1, a K-shell line intensity given by elemental fluorescent yield relative to oxygen and the elemental abundance relative to oxygen, and a $0.63 \mathrm{~h} \mathrm{ex-}$ posure time per field. The $\mathrm{O} K_{\alpha}$ line flux (quoted above) is based on the approximate luminosity measured for Europa in 1999 and 2000, assuming a height of $100 \mathrm{~km}$ above the moon's surface. Under these assumptions the count rate per image pixel in this line is $0.13 \mathrm{c} \mathrm{s}^{-1}$ while the background will be $5 \mathrm{c} \mathrm{s}^{-1}$ for thin shielding. The source $\mathrm{O} K_{\alpha}$ count rate is then about $4 \%$ of the background count rate. The resulting elemental abundances relative to oxygen (in percent) measurable at $3 \sigma$ from various elements are shown in Table 1 for spatial resolution elements $420 \mathrm{~m}$ on a side (ten detector pixels on a side). This spatial resolution is sufficient to carry out correlative measurements of surface composition with respect to known geologic structures on Europa and to identify regions of temporal change within the present upper limits from Voyager and Galileo observations of a few square kilometers over about twenty years (Phillips et al., 2000). With $0.63 \mathrm{~h}$ exposure time per field, in 30 days we would be able to measure 1140 fields to the sensitivities quoted in Table 1. Any continuum flux from the surface at the level of the instrumental background or higher would further reduce sensitivity. Taking this into account must await the completion of our modeling of $\mathrm{X}$-ray emission from the surface.

The trends in Table 1 arise because fluorescent yield increases, but effective collection area decreases, as atomic number increases. The abundance sensitivity scales inversely with the square root of observing time, and inversely with the square root of the number of detector pixels per spatial resolution element (or inversely with number of detector pixels along a side of a spatial resolution element). The proposed system has the flexibility to make the map resolution elements bigger if necessary depending on the line intensities actually found. Combining pixels will greatly improve sensitivity, at the expense of spatial resolution. The background at Europa is likely to be longitude dependent, with lower background, and thus higher resolution, on the leading hemisphere. This is because corotating magnetospheric field lines tend to be rapidly depleted of energetic electrons on ini- 
tial contact with the trailing hemisphere surface (Paranicas et al., 2001). On the other hand, sulfates and other nonice species are more diffusely mixed into the $\mathrm{H}_{2} \mathrm{O}$ ice matrix on the leading hemisphere, so abundances are lower than for fully exposed sulfates, etc., on the trailing hemisphere. In any case, one could expect to be able to provide abundances for the major elemental constituents of the surface and many of the minor constituents as well. The main purpose of the instrument concept described here is to map this composition to test the models of the surface that have been proposed and to specifically look for elemental ratios that might be suggestive of surface coupling to an ocean source.

In the 1999 and 2000 Chandra data, Ganymede emitted $\sim \frac{1}{2}$ the flux from Europa, while Callisto was undetected at a statistically significant level. For the assumed instrument, however, the background at Ganymede's orbit is an order of magnitude less than at Europa, and at Callisto's orbit at least two orders of magnitude less. As the instrument is background limited, one would therefore expect to be able to map the elemental composition of Ganymede's surface, and possibly Callisto's as well.

Assuming a background equal to that at Europa but a distance of $120 R_{\mathrm{J}}$ (a very conservative scenario), we estimate a signal-to-noise from Io's X-ray emission between $33 \sigma$ and $90 \sigma$ in an exposure of 1.77 day (Io's orbital and rotational period), depending on background. Thus one could fully expect to be able to provide abundances for Io integrated over the moon's surface, and be able to look for possible abundance changes due to volcanic activity.

Chandra and XMM-Newton observations of Jupiter in 2003 show that the auroral emission falls mostly below $1 \mathrm{keV}$. The observed spectrum is consistent with emission from $\mathrm{K}$ fluorescent lines from more than one species of highly ionized oxygen and either L fluorescent lines from highly ionized sulfur or $\mathrm{K}$ fluorescent lines from highly ionized carbon. Below $1 \mathrm{keV}$ the curve for effective collection area versus energy of the telescope modules described here is fairly flat. The power emitted by Jupiter's disk, northern aurora, and southern aurora were 2.3, 1.0, and $0.5 \mathrm{GW}$, respectively, in 2000 and about one-half that for the disk in 2003. Spreading the auroral emission uniformly over a $30 \times 30$ pixel area, at a distance of $20 R_{\mathrm{J}}$ from the planet, one could measure the X-ray flux per image resolution element with a signal-to-noise ratio of $40-60$, depending on the background rate, in an exposure time of $10 \mathrm{~min}$. These estimated values for signal-tonoise indicate that one would be able to do spatially and temporally resolved measurements of the flux and spectra from Jupiter's aurora. Finally X-ray emission from the feet of satellite flux tubes could also be searched for and, if discovered, studies with an imaging X-ray spectrometer. These types of measurements are impossible to make from an Earth-orbiting spacecraft for the foreseeable future.

\section{Conclusion}

The jovian system is a rich and diverse source of X-ray emission associated with magnetospheric interactions with Jupiter's polar atmosphere (the X-ray aurora), the Io plasma torus, and the Galilean satellites. The technique of imaging X-ray spectroscopy from within the jovian system offers unprecedented opportunities, not achievable from Earth orbiting spacecraft, for the study of Jupiter's X-ray aurora, the IPT, and the composition of the surfaces of the Galilean moons, with significant implications for magnetospheric processes and flows. By combination of orbital and remote observations we can expect to make great progress on understanding compositional relations between these major components of the jovian system. Although exciting progress has been made in understanding the composition and geology of these moons, measurements of the abundances of trace species are needed for the IPT and the icy moon surfaces. For instance, here we have shown that those models presently proposed for the surface composition can be tested with the measurements described here and that elemental composition could be spatially resolved for the material in the bands on Europa's surface where upwelling of material has been suggested to be occurring. Over time scales of years we would also be able to detect temporal transients in small-scale $(\mathrm{km})$ composition distributions indicative of subsurface and magnetospheric activity. One of the most difficult problems in moon surface composition is to separate contributions from external and internal sources (Johnson et al., 2004), but time correlation of X-ray derived composition to in situ and remote measurements of magnetospheric activity by X-ray and other (magnetic field, plasma, energetic particle, UV, neutral atom) instruments may allow this separation.

\section{Acknowledgments}

This report on techniques for spectroscopic X-ray imaging of the jovian system is based in part on our ongoing feasibility study of the X-ray Mapper of Icy Moon Elements (X-MIME) being supported by NASA 62-973-80 from NASA's High Capability Instruments for Planetary Exploration (HCIPE) program. Other sources of support for J.F. Cooper and R.E. Johnson include contract NASW02037 with the Planetary Atmospheres Program, and Cooper has been further supported through Raytheon by contracts NASW-99029 and NASW-02005 with the Jovian System Data Analysis Program. We also acknowledge very useful inputs on jovian system science objectives and JIMO capabilities from individual members of the Science Definition Team for JIMO as also published by Greeley et al. (2004). Finally we wish to thank referee Dr. Robert Carlson for an insightful and thought-provoking review, and for several useful suggestions. 


\section{References}

Bagenal, F., 1994. Empirical model of the Io plasma torus. J. Geophys. Res. 99 (A6), 11043-11062.

Bagenal, F., Shemansky, D.E., McNutt Jr., R.L., Schreier, R., Eviatar, A., 1992. The abundance of $\mathrm{O}^{++}$in the jovian magnetosphere. Geophys. Res. Lett. 19 (2), 79-82.

Bhardwaj, A., Gladstone, G.R., 2000. Auroral emissions of the giant planets. Rev. Geophys. 38, 295-353.

Bhardwaj, A., Branduardi-Raymont, G., Elsner, R.F., Gladstone, G.R Ramsay, G., Rodriguez, P., Soria, R., Waite, J.H., Cravens, T.E., 2005. Solar control on Jupiter's equatorial X-Ray emissions: 26-29 November 2003 XMM-Newton observation. Geophys. Res. Lett. 32, doi:10.1029/2004GL021497.

Branduardi-Raymont, G., Elsner, R.F., Gladstone, G.R., Ramsay, G., Rodriguez, P., Soria, R., Waite, J.H., 2004. First observation of Jupiter by XMM-Newton. Astron. Astrophys. 424, 331-337.

Brown, M.E., 2001. Potassium in Europa's atmosphere. Icarus 151, 190195.

Brown, M.E., Hill, R.E., 1996. Discovery of an extended sodium atmosphere around Europa. Nature 380, 229-231.

Bunce, E.J., Cowley, S.W.H., Yeoman, T.K., 2004. Jovian cusp processes: Implications for the polar aurora. J. Geophys. Res. 109 (A9), A09S13, doi:10.1029/2003JA010280.

Calvin, W.M., Clark, R.N., 1993. Spectral distinctions between the leading and trailing hemispheres of Callisto: New observations. Icarus 104, 6978

Calvin, W.M., Clark, R.N., Brown, R.H., Spencer, J.R., 1995. Spectra of the icy Galilean satellites from 0.2 to 5 micron: A compilation, new observations, and a recent summary. J. Geophys. Res. 100 (E9), 1904119048

Carlson, R.W., 1999. A tenuous carbon dioxide atmosphere on Jupiter's moon Callisto. Science 283, 820-821.

Carlson, R.W., 2001. Spatial distribution of carbon dioxide, hydrogen peroxide, and sulfuric acid on Europa. Bull. Amer. Astron. Soc. 33, 1125.

Carlson, R.W., Johnson, R.E., Anderson, M.S., 1999. Sulfuric acid on Europa and the radiolytic sulfur cycle. Science 286, 97-99.

Carlson, R.W, Anderson, M.S., Mehlman, R., Johnson, R.E., 2005. Distribution of hydrate on Europa: Furfher evidence for sulfuric acid hydrate. Icarus. In press.

Cooper, J.F., Johnson, R.E., Mauk, B.H., Garret, H.B., Gehrels, N., 2001 Energetic ion and electron radiation of the icy Galilean satellites. Icarus 149, 133-159.

Cravens, T.E., 1997. Comet Hyakutake X-ray source: Charge transfer of solar wind heavy ions. Geophys. Res. Lett. 24, 105-108.

Cravens, T.E., 2002. X-ray emission from comets. Science 296, 1042-1045.

Cravens, T.E., Waite, J.H., Gombosi, T.I., Lugaz, N., Gladstone, G.R., Mauk, B.H., MacDowall, R.J., 2003. Implications of jovian X-ray emission for magnetosphere-ionosphere coupling. J. Geophys. Res. 108 (A12), 25, doi:10.1029/2003JA010050.

Dalton, J.B., Mogul, R., Kagawa, H.K., Chan, S.L., Jamieson, C.S., 2003. Methodologies and techniques for detecting extraterrestrial (microbial) life. Astrobiology 3 (3), 505-529.

Domingue, D., Hendrix, A., 2005. A search for temporal variability in the surface chemistry of the icy Galilean satellites. Icarus 173, 50-65.

Domingue, D.L., Lane, A.L., 1998. IUE views Europa: Temporal variations in the UV. Geophys. Res. Lett. 25 (24), 4421-4424.

Domingue, D.L., Lane, A.L., Beyer, R.A., 1998. IUE's detection of tenuous $\mathrm{SO}_{2}$ frost on Ganymede and its rapid time variability. Geophys. Res. Lett. 25 (16), 3117-3120

Elsner, R.F., 15 colleagues, 2002. Discovery of soft X-ray emission from Io, Europa, and the Io plasma torus. Astrophys. J. 572, 1077-1082.

Elsner, R.F., Lugaz, N., Waite, J.H., Cravens, T.E., Gladstone, G.R., Ford, P., Grodent, D., Bhardwaj, A., MacDowall, R.J., Desch, M.D., Majeed, T., 2005. Simultaneous Chandra X-ray, HST ultraviolet, and Ulysses radio observations of Jupiter's aurora. J. Geophys. Res. 110, doi:10.1029/2004JA010717.
Fanale, F.P., Li, Y.-H., De Carlo, E., Farley, C., Sharma, S.K., Horton, K., 2001. An experimental estimate of Europa's "ocean" composition independent of Galileo orbital remote sensing. J. Geophys. Res. 106 (7), 14595-14600.

Gehrels, N., Stone, E.C., 1983. Energetic oxygen and sulfur ions in the jovian magnetosphere and their contribution to the auroral excitation. J. Geophys. Res. 88 (A7), 5537-5550.

Geiss, J., Gloeckler, G., Balsiger, H., Fisk, L.A., Galvin, A.B., Giliem, F., Hamilton, D.C., Ipavich, F.M., Livi, S., Mall, U., Ogilvie, K.W., von Steiger, R., Wilken, B., 1992. Plasma composition results in Jupiter's magnetosphere: Initial results from the Solar Wind Ion Composition Spectrometer. Science 257, 1535-1539.

Gladstone, G.R., Hall, D.T., 1998. Recent results from EUVE observations of the Io plasma torus and Jupiter. J. Geophys. Res. 103, 19927-19934.

Gladstone, G.R., Waite, J.H., Lewis, W.S., 1998. Secular and local time dependence of jovian X-ray emission. J. Geophys. Res. 103, 20083 20088.

Gladstone, G.R., Waite, J.H., Grodent, D., Lewis, W.S., Crary, F.J., Elsner, R.F., Weisskopf, M.C., Majeed, T., Jahn, J.M., Bhardwaj, A., Clarke, J.T., Young, D.T., 2002. A pulsating auroral X-ray hot spot on Jupiter. Nature 415, 1000-1003.

Greeley, R., Johnson, T., the JIMOS Science Definition Team, 2004. Report of the NASA Science Definition Team for the Jupiter icy moons orbiter (JIMO), NASA, released Feb. 13, 2004.

Hall, D.T., Gladstone, G.R., Moos, H.W., Bagenal, F., Clarke, J.T., Feldman, P.D., McGrath, M.A., Schneider, N.M., Shemansky, D.E., Strobel, D.F., Waite, J.H., 1994. Extreme ultraviolet explorer observation of Jupiter's Io plasma torus. Astrophys. J. Lett. 426, L51-L54.

Hamilton, D.C., Gloeckler, G., Krimigis, S.M., Lanzerotti, L.J., 1981. Composition of nonthermal ions in the jovian magnetosphere. J. Geophys. Res. 86, 8301-8318.

Hibbitts, C.A., McCord, T.B., Hansen, G.B., 2000. Distributions of $\mathrm{CO}_{2}$ and $\mathrm{SO}_{2}$ on the surface of Callisto. J. Geophys. Res. 105 (E9), 2254122557.

Johansson, S.A.E., Campbell, J.L., Malmquist, K.G., 1995. ParticleInduced X-Ray Emission Spectrometry (PIXE). Wiley, New York.

Johnson, R.E., 2000. Sodium at Europa. Icarus 143, 429-433.

Johnson, R.E., Quickenden, T.I., Cooper, P.D., McKinley, A.J., Freeman, C.G., 2003. The production of oxidants in Europa's surface. Astrobiology $3,823-850$

Johnson, R.E., Carlson, R.W., Cooper, J.F., Paranicas, C., Moore, H.M., Wong, M.C., 2004. Radiation effects on the surface of the Galilean satellites. In: Bagenal, F., Dowling, T., McKinnon, W.B. (Eds.), JupiterThe Planet, Satellites, and Magnetosphere. Cambridge Univ. Press, Cambridge, pp. 485-512.

Karanikola, I., Athanasiou, M., Anagnostopoulos, G.C., Pavlos, G.P., Preka-Papadema, P., 2004. Quasi-periodic emissions (15-80 min) from the poles of Jupiter as a principal source of the large-scale highlatitude magnetopause boundary layer of energetic particle. Planet. Space Sci. 52, 543-559.

Kargel, J.S., Kaye, J.Z., Head III, J.W., Marion, G.M., Sassen, R., Crowley, J.K., Ballesteros, O.P., Grant, S.A., Hogenboom, D.L., 2000. Europa's crust and ocean: Origin, composition, and the prospects for life. Icarus 148, 226-265.

Kivelson, M.G., Bagenal, F., Kurth, W.S., Neubauer, F.M., Paranicas, C., Saur, J., 2004. Magnetospheric interactions with satellites. In: Bagenal, F., Dowling, T., McKinnon, W.B. (Eds.), Jupiter-The Planet, Satellites, and Magnetosphere. Cambridge Univ. Press, Cambridge, pp. 513-536.

Krieger, B., Ewell, K., Ludewigt, B., Maier, M., Marković, D., Milgrome, O., Wang, Y.J., 2001. An $8 \times 8$ pixel array IC for X-ray spectroscopy. IEEE Trans. Nucl. Sci. NS48, 493-498.

Lane, A.L., Domingue, D.L., 1997. IUE's view of Callisto: Detection of an $\mathrm{SO}_{2}$ absorption correlated to possible torus neutral wind alterations. Geophys. Res. Lett. 24 (9), 1143-1146.

Leblanc, F., Johnson, R.E., Brown, M.E., 2002. Europa's sodium atmosphere: An ocean source? Icarus 159, 132-144. 
MacDowall, R.J., Kaiser, M.J., Desch, M.D., Farrell, W.M., Hess, R.A., Stone, R.G., 1993. Quasiperodic jovian radio bursts: Observations from the Ulysses radio and plasma wave experiment. Planet. Space Sci. 41, 1059-1072.

Mauk, B.H., Mitchell, D.G., Krimigis, S.M., Roelof, E.C., Paranicas, C.P., 2003. Energetic neutral atoms from a trans-Europa gas torus at Jupiter. Nature 421, 920-922.

Mauk, B.H., Mitchell, D.G., McEntire, R.W., Paranicas, C.P., Roelof, E.C., Williams, D.J., Krimigis, S.M., 2004. Energetic ion characteristics and neutral gas interactions in Jupiter's magnetosphere. J. Geophys. Res. 109, A09S12, doi:10.1029/2003JA010270.

Maurellis, A.N., Cravens, T.E., Gladstone, G.R., Waite, J.H., Acton, L.W., 2000. Jovian X-ray emission from solar X-ray scattering. Geophys. Res. Lett. 27, 1339-1342.

McCord, T.B., Hansen, G.B., Fanale, F.P., Carlson, R.W., Matson, D.L., Johnson, T.V., Smythe, W.D., Crowley, J.K., Martin, P.D., Ocampo, A., Hibbitts, C.A., Granahan, J.C., the NIMS Team, 1998a. Salts on Europa's surface detected by Galileo's Near Infrared Mapping Spectrometer. Science 280, 1242-1245.

McCord, T.B., Hansen, G.B., Clark, R.N., Martin, P.D., Hibbitts, C.A., Fanale, F.P., Granahan, J.C., Segura, M., Matson, D.L., Johnson, T.V., Carlson, R.W., Smythe, W.D., Danielson, G.E., the NIMS Team, 1998b. Nonwater ice constituents in the surface material of the icy Galilean satellites from the Galileo near-infrared mapping spectrometer investigation. J. Geophys. Res. 103 (E4), 8603-8626.

McCord, T.B., Hansen, G.B., Matson, D.L., Johnson, T.V., Crowley, J.K., Fanale, F.P., Carlson, R.W., Smythe, W.D., Martin, P.D., Hibbitts, C.A., Granahan, J.C., Ocampo, A., 1999. Hydrated salt minerals on Europa's surface from the Galileo near-infrared mapping spectrometer (NIMS) investigation. J. Geophys. Res. 104 (E5), 11827-11851.

McGrath, M.A., Lellouch, E., Strobel, D.F., Feldman, P.D., Johnson, R.E., 2004. Satellite atmospheres. In: Bagenal, F., McKinnon, W., Dowling, T. (Eds.), Jupiter-The Planet, Satellites, and Magnetosphere. Cambridge Univ. Press, Cambridge, pp. 457-483.

McKibben, R.B., Simpson, J.A., Zhang, M., 1993. Impulsive bursts of relative electrons discovered during Ulysses traversal of Jupiter's dusk-side magnetosphere. Planet. Space Sci. 41, 1041-1058.

McKinnon, W.B., Zolensky, M.E., 2003. Sulfate content of Europa's ocean and shell: Evolutionary considerations and some geological and astrobiological implications. Astrobiology 3 (4), 879-897.

Metzger, A.E., Gilman, D.A., Luthey, L., Hurley, K.C., Schnopper, H.W., 1983. The detection of X-rays from Jupiter. J. Geophys. Res. 88, 77317741.

Miyata, E., Kouno, H., Kamazuka, T., Tomoyuki, F., Mihara, M., Matsuta, K., Tanaka, K., Tsunemi, H., Minamisono, T., Tomida, H., Miyaguchi, K., 2003. Radiation damage test of the X-ray CCDs for MAXI onboard the International Space Station. In: Proc. SPIE, vol. 4851, pp. 10801091.
Paranicas, C., Paterson, W.R., Cheng, A.F., Mauk, B.H., McEntire, R.W., Frank, L.A., Williams, D.J., 1999. Energetic particle observations near Ganymede. J. Geophys. Res. 104, 17459-17470.

Paranicas, C., Carlson, R.W., Johnson, R.E., 2001. Electron bombardment of Europa. Geophys. Res. Lett. 28, 673-676.

Paranicas, C., Ratliff, J.M., Mauk, B.H., Cohen, C., Johnson, R.E., 2002a. The ion environment near Europa and its role in surface energetics. Geophys. Res. Lett. 29, 18, doi:10.1029/2001GL0147127.

Paranicas, C.P.M., Volwerk, M., Kivelson, M.G., 2002b. Flow Diversion at Europa. Eos Trans. AGU, 83 (19), Spring Sci. Meet. Suppl. Abstract P21B-07.

Phillips, C.B., McEwen, A.S., Hoppes, G.V., Fagents, S.A., Greeley, R., Klemaszewski, J.E., Pappalardo, R.T., Klaasen, K.P., Breneman, H.H., 2000. The search for current geologic activity on Europa. J. Geophys. Res. 105 (E9), 22579-22597.

Pierazzo, E., Chyba, C.F., 2002. Cometary delivery of biogenic elements to Europa. Icarus 157, 120-127.

Pospieszalska, M.K., Johnson, R.E., 1989. Magnetospheric ion bombardment profiles of satellites-Europa and Dione. Icarus 78, 1-13.

Rybicki, G.B., Lightman, A.P., 1979. Radiative Processes in Astrophysics. Wiley, New York.

Saur, J., Strobel, D.F., Neubauer, F.M., 1998. Interaction of the jovian magnetosphere with Europa: Constraints on the neutral atmosphere. J. Geophys. Res. 103 (E9), 19947-19962.

Shematovich, V.I., Johnson, R.E., Cooper, J.F., Wong, M.C., 2005. Surfacebounded atmosphere of Europa. Icarus 173 (2), 480-498.

Struder, L., 2000. High-resolution imaging X-ray spectrometers. Nucl. Instrum. Methods 454, 73-113.

Smythe, W.D., Carlson, R.W., Ocampo, A., Matson, D., Johnson, T.V., McCord, T.B., Hansen, G.E., Soderblom, L.A., Clark, R.N., 1998. Absorption bands in the spectrum of Europa detected by the Galileo NIMS instrument. Lunar Planet. Sci. XXIX. Abtract 1532.

Tucker, W.H., 1975. Radiation Processes in Astrophysics. MIT Press, Cambridge, MA.

Waite Jr., J.H., Bagenal, F., Seward, F., Na, C., Gladstone, G.R., Cravens, T.E., Hurley, K., Clarke, J.T., Elsner, R.F., Stern, S.A., 1994. ROSAT observations of the Jupiter aurora. J. Geophys. Res. 99, 14799-14809.

Waite, J.H., Gladstone, G.R., Lewis, W.S., Drossart, P., Cravens, T.E., Maurellis, A.N., Mauk, B., Miller, S., 1997. Equatorial X-ray emissions: Implications for Jupiter's high exospheric temperature. Science 276, 104-108.

Waite, J.H., Gladstone, G.R., Lewis, W.S., Goldstein, R., McComas, D.J., Riley, P., Walker, R.J., Robertson, P., Desai, S., Clarke, J., Young, D.T., 2001. An auroral flare at Jupiter. Nature 4 (10), 787-789.

Ziegler, J.F., Biersack, J.P., Littmark, U., 1985. The Stopping and Range of Ions in Solids. Pergamon, New York, NY.

Zolotov, M.Y., Shock, E.L., 2001. Composition and stability of salts on the surface of Europa and their oceanic origin. J. Geophys. Res. 106 (E12), 32815-32827. 